\title{
Influence of Supporting Cells on Neuronal Degeneration After Hair Cell Loss
}

\author{
Mitsuru Sugawara, ${ }^{1,2,3}$ Gabriel Corfas, ${ }^{4,5}$ and M. Charles Liberman ${ }^{1,2}$ \\ ${ }^{1}$ Eaton-Peabody Laboratory, Massachusetts Eye and Ear Infirmary, Boston, MA 02114, USA \\ ${ }^{2}$ Department of Otology and Laryngology, Harvard Medical School, Boston, MA 02114, USA \\ ${ }^{3}$ Department of Otolaryngology, Tohoku University School of Medicine, Sendai, Japan \\ ${ }^{4}$ Division of Neuroscience, Children's Hospital, Boston, MA 02115, USA \\ ${ }^{5}$ Department of Neurology, Harvard Medical School, Boston, MA 02115, USA
}

Received: 23 August 2005; Accepted: 15 December 2004; Online publication: 10 June 2005

\begin{abstract}
In sensorineural hearing loss, hair cell loss is often followed by loss of cochlear nerve fibers, which can continue for years after the insult. The degree and time course of neuronal loss varies, but the reasons for this variation are unclear. The present study addresses this issue with a quantitative analysis of hair cell, supporting cell, and neuronal survival in animals with long-term survival of up to 5.5 years from two types of drug-induced hair cell loss: aminoglycoside antibiotics and platinum-containing chemotherapeutics. To complement the analysis of the effects of organ of Corti damage on neuronal survival, cases of primary neuronal degeneration, via auditory nerve section, are also assessed. Analysis shows that (1) long-term neuronal survival is enhanced when supporting cells in the inner hair cell (IHC) area remain intact; (2) after hair cell loss, the time course of neuronal loss is slower in the apex than in the base; (3) primary loss of cochlear nerve fibers does not lead to secondary degeneration of sensory cells or supporting cells in the organ of Corti; and (4) after auditory nerve section, there can be a massive reinnervation of the IHC region, especially in the apex. Results are consistent with the idea that supporting cells participate in the regulation of neuronal survival and neuronal sprouting in the organ of Corti.
\end{abstract}

Correspondence to: M. Charles Liberman - Eaton-Peabody Laboratory - Massachusetts Eye and Ear Infirmary $\cdot 243$ Charles Street, Boston, MA 02114, USA. Telephone: (617) 573-3745; fax: (617) 720-4408; email: mcl@epl.meei.harvard.edu
Keywords: cochlea, sensorineural hearing loss, spiral ganglion

\section{INTRODUCTION}

Cochlear hair cells in the mammalian inner ear are connected to the brain by two types of bipolar sensory neurons. Both have cell bodies in the spiral ganglion and send peripheral axons to hair cells and central axons to the cochlear nucleus (Brown et al. 1988; Fekete et al. 1982; Kiang et al. 1982a). In sensorineural hearing loss, hair cells are often a primary site of damage (Schuknecht 1974). After hair cell loss, surviving cochlear nerve fibers can be stimulated with a cochlear implant. However, these nerve fibers undergo secondary degeneration (Spoendlin 1984), which can progress for many years (Johnsson 1974a; Leake and Hradek 1988). Such neuronal degeneration is particularly severe in cochlear regions where inner hair cells (IHCs) are destroyed (Johnsson 1974a; Leake and Hradek 1988; Zimmermann et al. 1995), suggesting an important role for IHCs in promoting neuronal survival. However, even when the hair cell loss is complete, the degree and time course of neuronal loss are variable. Histopathological studies have suggested that supporting cell survival is an important factor (e.g., Suzuka and Schuknecht 1988), but quantitative data are lacking.

Gene expression studies support the idea that cochlear-supporting cells are important to neuronal survival. Supporting cells ensheath the unmyelinated process of cochlear nerve fibers within the organ of 
Corti and express many of the same markers as neuron-ensheathing glial cells from the central nervous system (Rio et al. 2002). Glial cells provide trophic support necessary for neuronal survival under normal conditions and after injury (Cui et al. 2001; Reddy et al. 2003). Furthermore, interruption of a signaling pathway in supporting cells in the mouse inner ear leads to massive loss of type-I ganglion cells, without concomitant loss of IHCs, as well as reduced expression of a neurotrophin (NT-3) (Stankovic et al. 2004), known to be critical for neuronal survival in the cochlea (Miller et al. 1997).

To examine empirically the role of cochlear-supporting cells in long-term neuronal survival, we studied the quantitative relationship between supporting cell survival and the survival of nerve fibers after massive IHC loss resulting from carboplatin treatment (in chinchillas) or aminoglycoside treatment (in cats). Carboplatin-treated chinchilla ears are interesting because cell loss in the organ of Corti is restricted to IHCs; the aminoglycoside-treated ears are useful because, in addition to complete loss of IHC, there is a variable supporting cell loss, thus allowing a separation of the effects of degeneration in the two cell classes. To complement the analysis of secondary neuronal degeneration, we also studied cases in which the auditory nerve had been sectioned, leading to massive loss of type-I ganglion cells. While less common, primary neuronal degeneration can also underlie human deafness (Merchant et al. 2001; Starr et al. 1996).

The ears studied, with survival of up to 5.5-year posttreatment, were generated in the course of previous studies with a variety of aims; thus, a variety of histological techniques were used. Nevertheless, given that animal studies with survival times exceeding 1 year are rare and material such as this is difficult to generate, the quantitative analysis presented here provides rare insight into long-term neuronal sequelae of cell loss in the organ of Corti and the cochlear cellular sequelae of long-term neuronal loss.

The data show that (1) long-term neuronal survival is enhanced when supporting cells in the IHC area remain intact; (2) after hair cell loss, the time course of neuronal loss is slower in the apex than in the base; and (3) primary loss of cochlear nerve fibers does not lead to secondary degeneration of sensory cells or supporting cells in the organ of Corti, rather there is reinnervation of the IHC region, especially in the apex.

\section{METHODS}

\section{Animals and drug or surgical treatments}

Cats treated with aminoglycosides ( $n=4$ animals; $n=$ 8 cochleas) were given subcutaneous injections of kanamycin (200 mg/kg) for 9-12 days. Cats in which the auditory nerve was cut $(n=11)$ were anesthetized with sodium pentobarbital, after which the auditory nerve was exposed from a posterior fossa approach after skull removal and cerebellar retraction. The auditory portion of the VIIIth nerve was cut with a microknife, taking care to spare the facial nerve which also runs in the internal auditory meatus. Chinchillas ( $n=9$ animals; $n=18$ cochleas) were anesthetized with ketamine/xylazine and infused intravenously with carboplatin over 5-10 min at a dosage of $400-500 \mathrm{mg} / \mathrm{m}^{2}$. For the study of drug treatments, control chinchillas ( $n=2$ animals; $n=4$ cochleas) and control cats $(n=2$ animals; $n=4$ cochleas) received no injections. For the cut-nerve experiments, the opposite (nonsectioned) ears served as controls. All animals were housed in the animal care facility until sacrifice.

\section{Histological processing}

Cats were perfused intravascularly with either mixed aldehydes (for animals with survival times $<6$ months) or Heidenhain-Susa solution (for survival times $>6$ months). The mixed aldehyde fixative consisted of $2.5 \%$ glutaraldehyde and $1.25 \%$ paraformaldehyde in $0.1 \mathrm{M}$ phosphate buffer. After perfusion and dissection, the tissue was postfixed in the same solution for $24 \mathrm{~h}$ at $4^{\circ} \mathrm{C}$. Heidenhain-Susa fixative was prepared as described in detail elsewhere (Schuknecht 1974). After perfusion and dissection, the tissue was postfixed in the same solution for 24 $\mathrm{h}$ at $4^{\circ} \mathrm{C}$. After fixation, both temporal bones were extracted, decalcified, dehydrated, and embedded in either paraffin (survivals $<6$ months) or celloidin (survivals $>6$ months) and were sectioned at $20 \mu \mathrm{m}$. Every 10th section was mounted on slides and stained with hematoxylin and eosin. Chinchillas were perfused with mixed aldehydes in phosphate buffer. Temporal bones were extracted, postfixed in osmium, and then dehydrated and embedded in plastic. Plastic-embedded cochleas from animals with 2- to 4month postcarboplatin survivals were processed as surface preparations. Cochleas from animals with 5.5year survivals were sectioned at $40 \mu \mathrm{m}$ and every section mounted on slides.

\section{Histological analysis}

Cochlear length reconstruction. For sectioned ears, the cochlear spiral was reconstructed in three dimensions using Neurolucida software (Microbrightfield, Colchester, VT). Total cochlear length was determined as well as the distance from base represented 
by each area within each section, using custom LabVIEW (National Instruments) software to which the Neurolucida 3-D coordinates were ported. For surface preparations, cochlear lengths were measured directly from the whole mount by computerized planimetry. Cochlear location was converted to frequency according to published maps for cat (Liberman 1982a) or chinchilla (Eldredge et al. 1981).

Hair cell and supporting cell counts. For all cochlear material, the fractional survival of hair cells and supporting cells was determined using an oil immersion lens (N.A. 1.3) with Nomarski optics. In sectioned material, all cells were examined throughout the entire section thickness and accurate determinations of fractional loss of each cell type were made. In surface preparations, all hair cell and pillar cell populations were evaluated and quantified throughout the cochlear spiral.

Neuronal counts. Estimates or counts of neuronal loss focused on the peripheral axons in the osseous spiral lamina. Fibers counted via this approach represent mostly type-I afferents contacting IHCs, since (1) OHC afferents are unmyelinated and not visible in the light microscope (Kiang et al. 1982a) and (2) OHC efferents, although myelinated, number $<1000$ per cochlea (Warr 1975) compared to $\sim 50,000$ type-I afferents per normal cochlea (Gacek and Rasmussen 1961). Type-I neurons generally take a direct radial course from the ganglion region to the nearest IHC area, where the vast majority contact a single IHC via a single terminal swelling (Liberman 1982b).

(1) Carboplatin cases (chinchilla): In all chinchilla cochleas, all of which were osmium-stained, neuronal survival was determined by counting myelinated peripheral axons in tangential sections through the osseous spiral lamina. For those cases in which the cochlea was serially sectioned (survivals $>1$ year), as schematized in Figure 1A, the correct orientation is achieved at six different locations along the cochlear spiral. At each location, the section just distal to the edge of the limbus was chosen for quantitative analysis, as schematized in Figure 1B. For this section, all myelinated fibers were counted within a field of view corresponding to $\sim 340 \mu \mathrm{m}$ of cochlear length, using an oil-immersion lens (N.A. 1.3) with Nomarski optics. For those cases in which the cochlea was initially processed as a surface preparation (survivals $<1$ year), after analysis of hair cells and supporting cells was completed, pieces of the cochlear spiral corresponding to the six locations schematized in Figure 1A were reembedded and sectioned in the orientation indicated in Figure 1B (after which point the neuronal analysis was carried out as described above). Normative data on neuronal density (panel C) were obtained from four normal cochleas; data from drug-treated ears were expressed relative to the mean

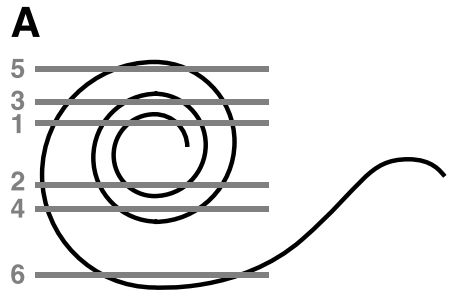

B

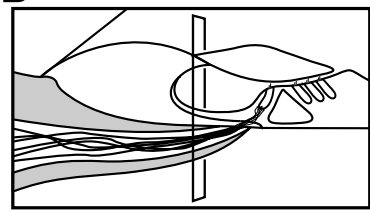

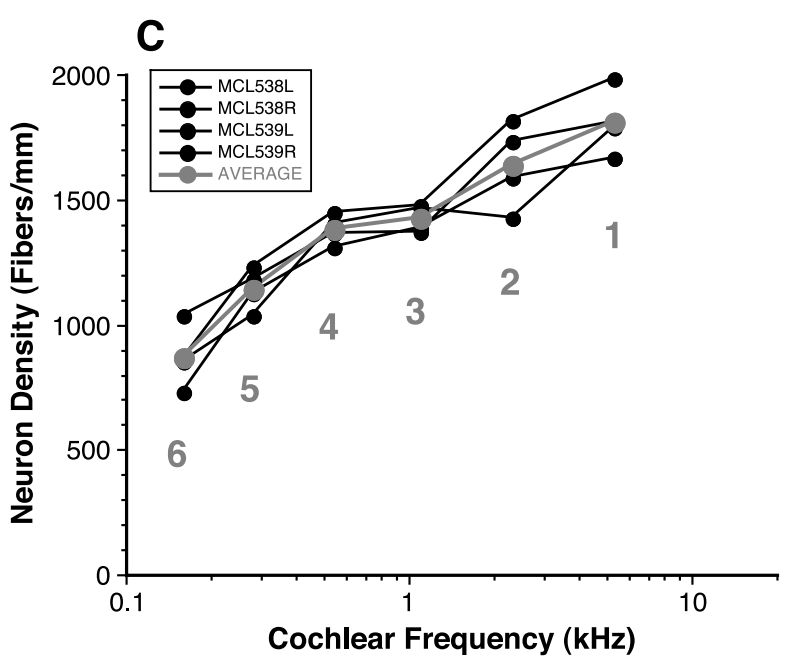

relative to the organ Corti. C Nerve fiber densities for each of four normal cases (black symbols) at each of the six sample locations are plotted along with the average value at each locus (gray symbols). Numbers below each gray symbol refer to the locations schematized

in $\mathbf{A}$.
FIG. 1. Density of myelinated axons in the osseous spiral lamina in four normal chinchillas. A Schematic two-dimensional projection of the cochlea showing the relative positions of the six sections selected from each ear in which peripheral axons were counted; that is, the sections through each half turn in which the cochlear spiral is cut tangentially. B The plane of section and its position 
normal value for the appropriate cochlear location.

(2) Aminoglycoside and cut-nerve cases (cat): In cat cochleas, the histological preparation (lacking the osmium postfixation) did not always provide single-fiber resolution; thus, neuronal density in the osseous spiral lamina was estimated using a visual rating scale. As can be seen by the micrographs in Figure 2, although single fibers can be resolved only when the neuronal density is greatly reduced (i.e., Fig. 2C), it is possible,
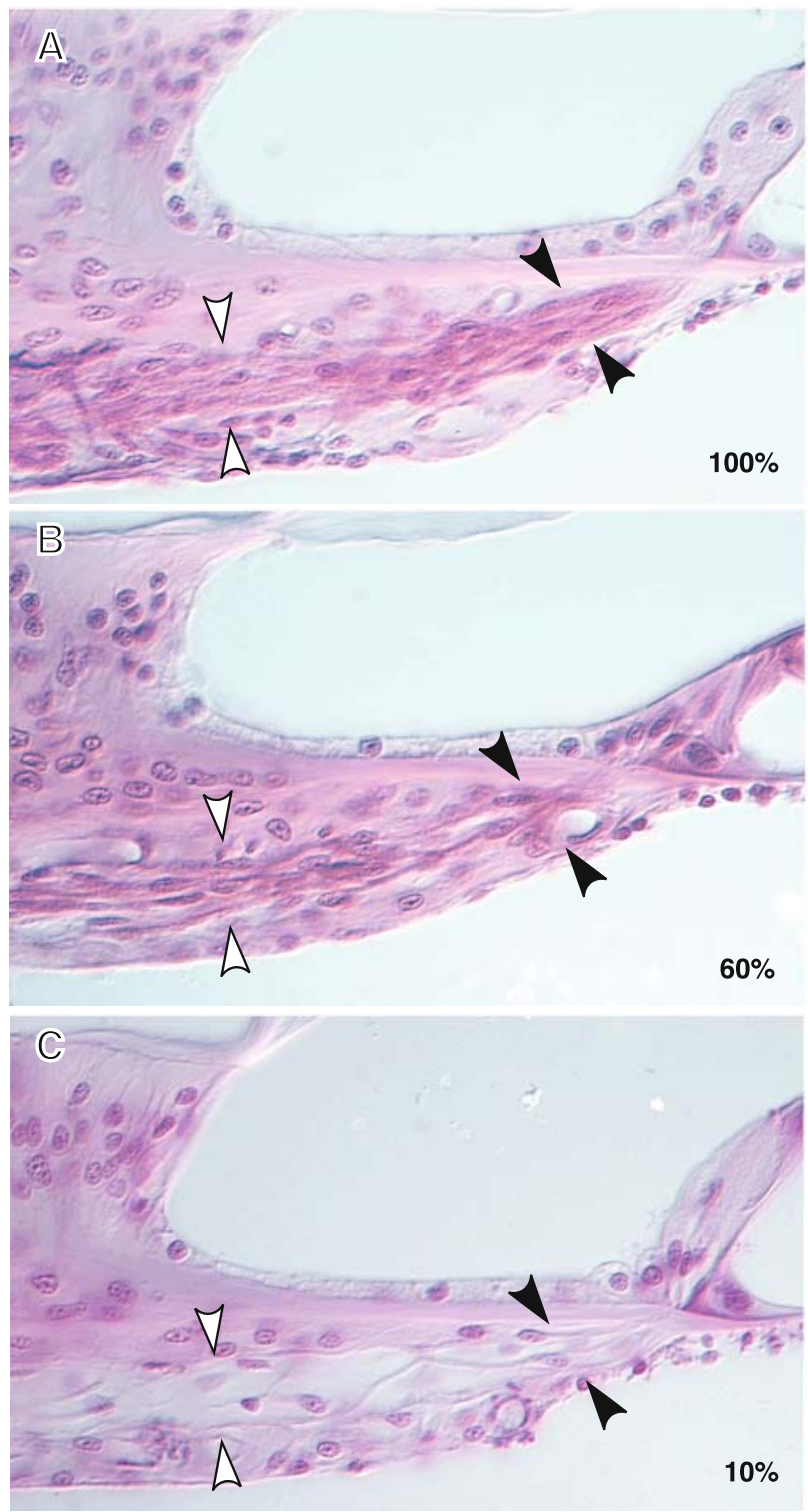

FIG. 2. Photomicrographs of sections through the osseous spiral lamina from the apical turn of three different cats, selected to illustrate the visual rating scale used to assess fiber density. In each panel, fiber density estimate assigned by the observer is indicated in the lower right corner. The pairs of arrowheads span the region of the spiral lamina which was included in the visual analysis. A Normal cat, B 1.5-year survival from kanamycin treatment, C 3.1year survival from kanamycin treatment. nevertheless, to estimate the extent to which the bundles of peripheral axons fill the interosseous spaces within the spiral lamina in all cases. With experience, different observers arrive independently at similar ratings for the same region. Estimates of neuronal density were made in every cochlear region within every mounted section. The observer estimated the fractional survival $(0-100 \%)$ by comparison to location-matched regions from identically processed tissue from control animals. To minimize criterion drift, all neuronal estimation was carried out during a concentrated time period over a few consecutive days. The accuracy of this visual estimation technique was assessed in the following way: neuronal survival in the sectioned chinchilla cochleas was initially assessed in all sections by the same visual estimation technique. Subsequently, the tangential sections were reanalyzed quantitatively (where individual axons can be resolved): the degree of agreement between the two estimates was acceptable $\left(r^{2}=0.65\right)$.

\section{RESULTS}

The carboplatin model: selective hair cell loss with supporting cells intact

Chinchillas treated with i.v. carboplatin, a chemotherapeutic agent, routinely show an auditory neuropathy phenotype, i.e., cochlear neural responses, such as CAP or wave 1 of the ABR, are attenuated or absent, yet otoacoustic emissions, e.g., distortion products, remain normal (Liberman et al. 1997; Takeno et al. 1994).

As illustrated by photomicrographs in Figure 3 (compare panels A and D), the treated cochlea shows selective loss of IHCs. The overall architecture of the organ of Corti remains remarkably normal, even when examined at high magnification (Fig. 3B and E). Even 5.5 years after treatment, the supporting cells in the IHC area remain visible (Fig. 3E vs. B), including inner border (black arrow), inner phalangeal (white arrow), and inner pillar (gray arrow) cells, although nuclear positions may have shifted. In normal material, stereocilia are visible, making identification of IHCs unambiguous (white arrow in A). The loss of neurons is significant in this longsurviving case: panels $\mathrm{C}$ and $\mathrm{F}$ show micrographs of tangential sections through the osseous spiral lamina, where the myelinated peripheral axons of the type-I cochlear nerve fiber course from the organ of Corti towards the cell bodies in the spiral ganglion. In all cases, loss of spiral ganglion cells and their central axons appeared comparable to the loss of peripheral axons in the osseous spiral lamina. 

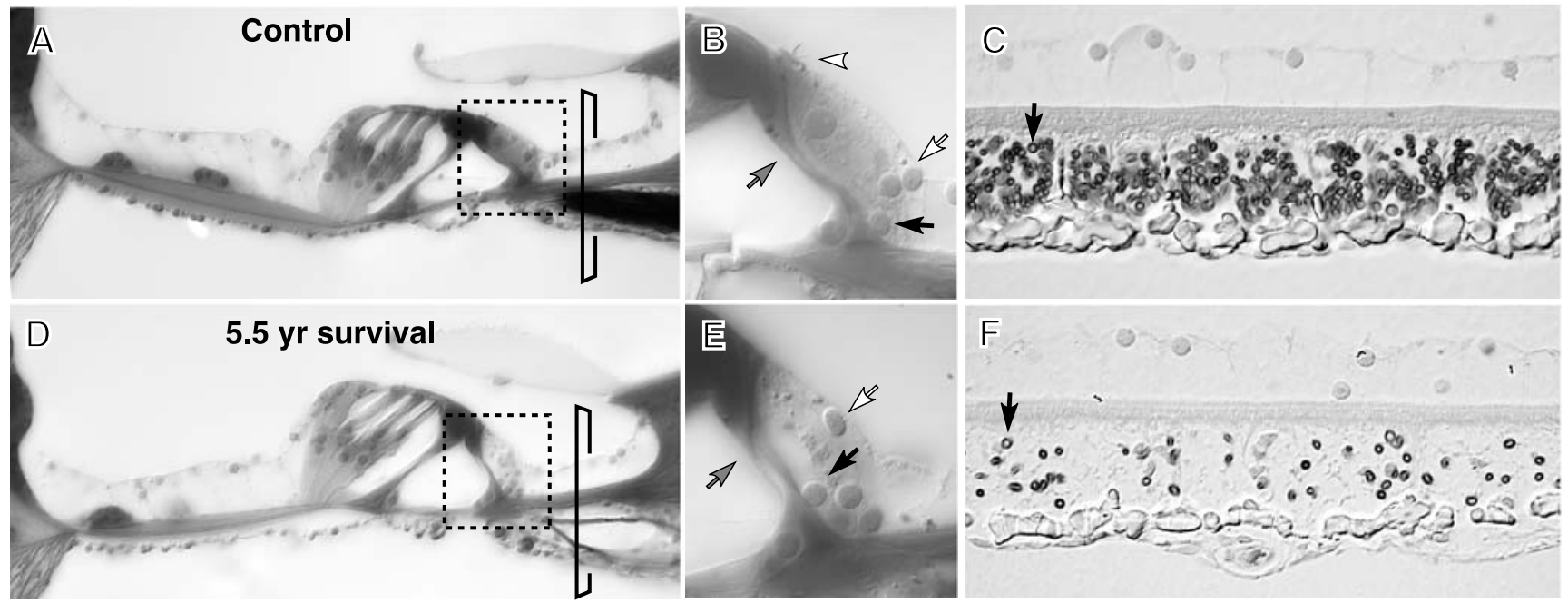

FIG. 3. Long-survival carboplatin-treated chinchillas show selective loss of inner hair cells $(\mathbf{D}, \mathbf{E})$ compared to normal ears $(\mathbf{A}, \mathbf{B})$ as well as loss of the peripheral axons of auditory nerve fibers from the osseous spiral lamina (C, F). B, E Higher magnifications of the dotted regions in $\mathbf{A}$ and $\mathbf{D}$, respectively. White-filled arrows point to nuclei of inner phalangeal cells, black arrows point to nuclei of inner

To analyze the histopathology more quantitatively, hair cells, supporting cells, and neurons were counted (see "Methods") along the cochlear spiral in each of four control and 15 carboplatin-treated cochleas: 10 with survival of a few months (74-132 days) and five with survival of 5.5 years. Representative cochleograms are shown in Figure 4. This quantitative analysis of peripheral axons along the cochlear spiral showed that neuronal survival can be border cells, gray-filled arrows point to the bodies of inner pillar cells. C, F From tangential sections through the osseous spiral lamina as schematized by the simulated section planes in $\mathbf{A}$ and $\mathbf{D}$ : black arrows point to individual myelinated fibers cut in cross-section. All images are from the upper basal turn.

significant without IHCs, especially in the cochlear apex.

At 2- to 4-month survivals, IHC loss was $>75 \%$ in all cochlear regions, OHC loss was minimal, and there was essentially no loss of supporting cells in the inner or outer hair cell areas (supporting cell data is not shown). Significant neuronal loss was already evident (Fig. 4A, B). However, in apical cochlear regions, neuronal loss was minimal despite total loss of IHCs.
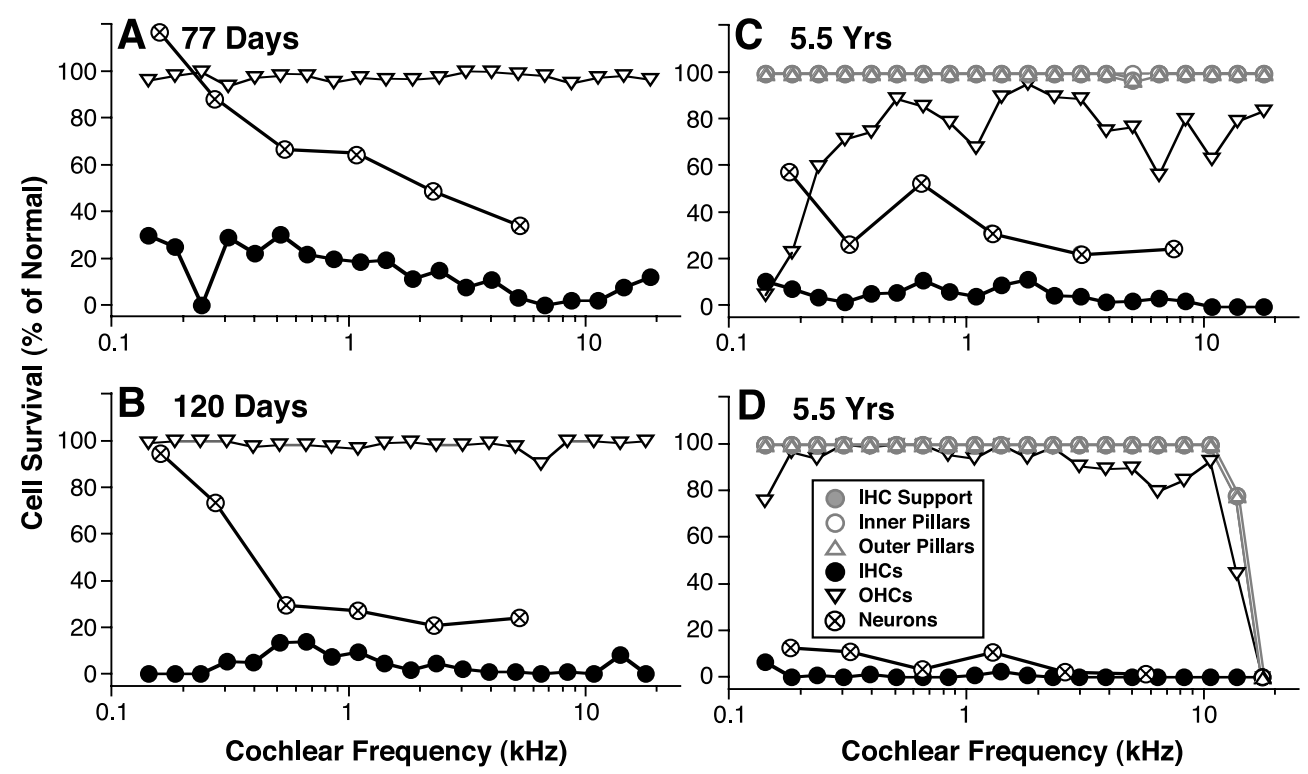

D 5.5 Yrs

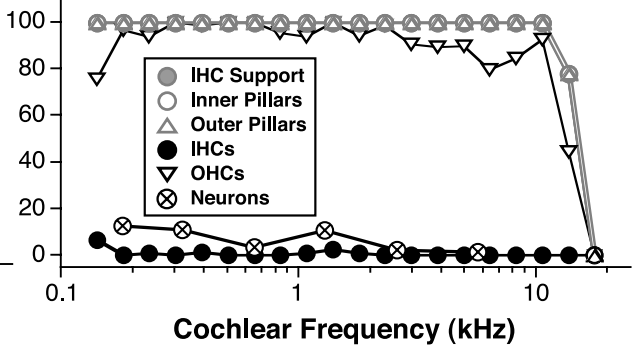

FIG. 4. Patterns of hair cell, supporting cell, and neuronal degeneration in four carboplatin-treated chinchillas: two with relatively short survival (A, B) and two with relatively long survival (C, D). Symbol key in D applies to all panels. Hair cell and

supporting cell data are averaged over bins corresponding to $5 \%$ of cochlear length. Neuronal counts are made in the six regions indicated in Figure $1 \mathrm{~A}$ and are expressed relative to the average data from normal ears shown in Figure 1C. 


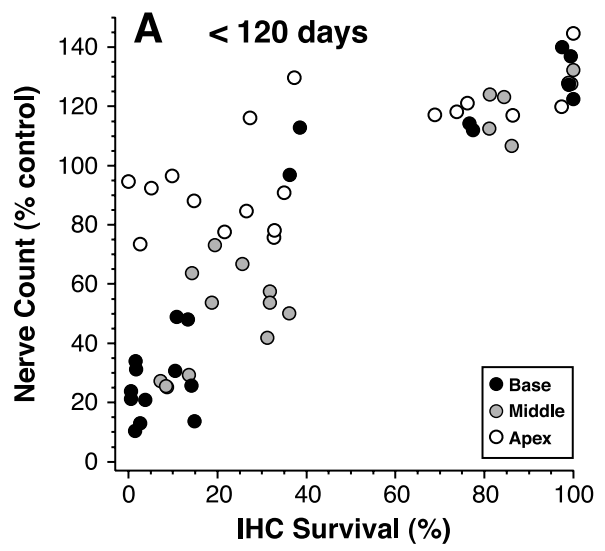

FIG. 5. Correlation between inner hair cell survival and neuronal survival for all carboplatin-treated ears, shown separately for shortsurvival (A, $n=10$ cochleas) and long-survival cases (B, $n=5$ cochleas). Data on these graphs compare the estimated fractional neuronal survival for one cochlear region of one ear to the value of $\mathrm{IHC}$ loss for the same cochlear region of the same ear (both values

At 5.5 years, the IHC loss was $>90 \%$ at all cochlear locations. OHC loss was $<25 \%$ in most regions, and there was essentially no loss of supporting cells in either the IHC or OHC areas (only the IHC area is shown in the plots). At this longer survival, the loss of cochlear neurons was more dramatic $(\mathrm{C}, \mathrm{D})$. However, a relatively small percentage of remaining IHCs was associated with larger fractional survival of neurons, especially in the cochlear apex.

The quantitative relation between fractional IHC loss and fractional neuronal loss is more clearly demonstrated in the scatterplots in Figure 5, which compare fractional IHC survival to fractional neuronal survival for all cochlear regions of all cases examined: 2- to 4-month posttreatment survival (panel A) vs. 5.5-year survival (panel B). Each data point represents mean values averaged over bins of $10 \%$ of cochlear length. In the apical turn (regions 5 and 6 in Fig. 1), neuronal loss at 2-4 months never exceeded $30 \%$, even in cochlear regions where IHC loss was complete (white-filled circles in panel A). In middle and basal turns (gray- and black-filled circles in panel A), neuronal survival was lower for the same degree of IHC survival. For long survival times, the fractional loss of IHCs was about threefold more than the fractional loss of neurons (see best fit straight line in panel B). For shorter survivals (panel A), a similar relation holds for basal and middle-turn data (for points with $>40 \%$ IHC survival, the neuronal survival has saturated at $\sim 120 \%$ and can climb no further ${ }^{1}$ ).

\footnotetext{
Maximal neuronal densities in the treated ears appear systematically higher than normal (by 20-40\%) in regions where most IHCs remain. Reasons for this are unclear, as control animals were of similar age and the same sex as the treated animals. The possibility that the carboplatin treatment led to neuronal sprouting has to be considered, but more data are needed.
}

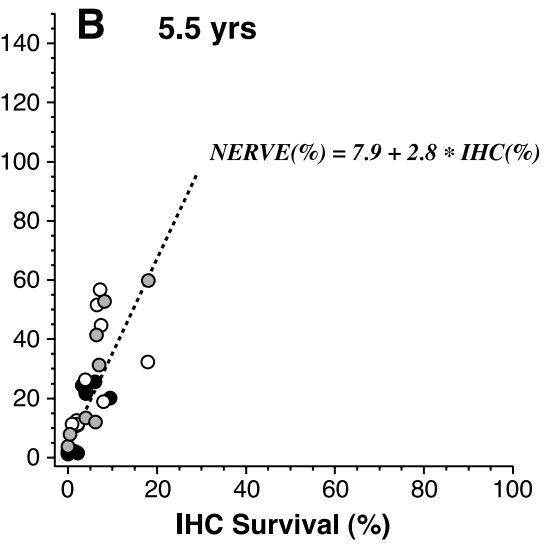

computed as described in Fig. 3). Data from the cochlear base, middle or apex are plotted with different symbols (see key) and correspond to regions in Figure $1 \mathrm{~A}$ labeled 1 and $2 ; 3$, and 4 ; or 5 and 6, respectively. Dashed line in $\mathbf{B}$ represents the best fit straight line; the equation describes the slope and intercept of that line.

For apical turn points, at shorter survivals, the slope is much shallower, but this is because $0 \%$ IHC survival appears to support $\sim 80 \%$ neuronal survival. The possibility that supporting cell survival is key to this enhanced neuronal survival is tested, in the next section, with data from aminoglycoside-treated cats.

Aminogylcoside treatment-mixed hair cell and supporting cell losses

High doses of aminoglycoside antibiotics such as kanamycin or neomycin, can destroy OHCs and IHCs throughout the cochlea. In some regions, especially in the apical half of the cochlea, supporting cells remain after hair cells are destroyed, and the organ of Corti architecture is remarkably normal. For example, apical-turn micrographs in Figure 6D and F show that inner border cells (black arrows), inner phalangeal cells (white arrows) and inner pillar cells (gray arrows) remain intact, even 3.1-year posttreatment. Aminoglycoside-treated ears also show cochlear regions in which all hair cells and supporting cells are destroyed. For example, the images in Figure $6 \mathrm{G}$ and $\mathrm{H}$ are from nearby cochlear regions in the same ear: both show hair cell loss, but in one (6G) supporting cells are intact, whereas in the other region $(6 \mathrm{H})$, all supporting cells are gone. Note the enhanced neuronal survival (black arrows point to the ganglion cells), in the region where supporting cells remain.

These examples suggest that, when IHCs are lost via aminoglycosides, supporting cell survival may enhance neuronal survival for several years posttreatment, especially in the apex. In aminoglycoside ears, the role of supporting cells in enhancing neuronal 


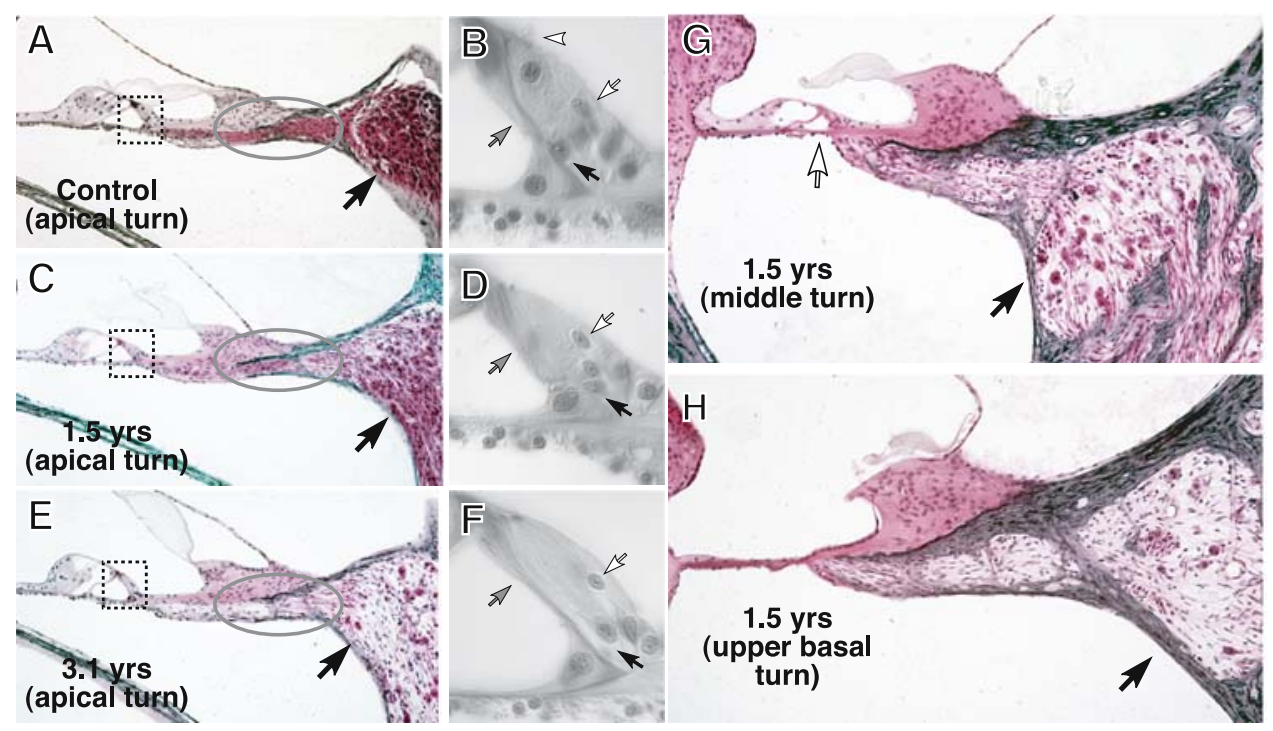

FIG. 6. Photomicrographs illustrating the selective loss of hair cells in kanamycin-treated ears $(\mathbf{C}, \mathbf{D}, \mathbf{E}, \mathbf{F}, \mathbf{G})$ and the improved neuronal survival compared to regions in which supporting cells of the organ of Corti are lost as well $(\mathbf{H})$. Black arrows in $\mathbf{A}, \mathbf{C}, \mathbf{E}, \mathbf{G}$, and $\mathbf{H}$ point to the ganglion cell region. $\mathbf{A}, \mathbf{C}$, and $\mathbf{E}$ Comparison of neuronal survival in the apical turn of control ear with two long-surviving kanamycin ears. Gray ellipses surround peripheral axons in the osseous spiral lamina. B, D, and F. High-power views of the IHC

survival can be assessed independently of the IHCs, because there is a variable degree of supporting cell survival in regions of total hair cell loss. To investigate these relations, hair cells, supporting cells and neurons were counted in both ears from four aminoglycoside-treated cats with survival times of 1.5, 3.1, 4.1 , and 5.5 years. Two representative cases are shown in Figure 7: in both, almost all IHCs and OHCs were destroyed, yet most IHC-supporting cells remain in the apical half of the cochlea. In the 1.5-year survival case (Fig. 7A), there is significantly more neuronal survival in the cochlear region where supporting cells remain. Furthermore, the edge of the supporting cell areas shown in dotted squares of $\mathbf{A}, \mathbf{C}$, and $\mathbf{E}$, respectively. Black and white arrows indicate nuclei of IHC-supporting cells; gray arrows point to inner pillar cells, and white arrowhead (B only) indicates the stereocilia bundle on an IHC. G, H Low-power micrographs of the cochlear duct in two adjacent half turns from the same kanamycin ear: locations of these views are indicated on the cytocochleogram in Figure 6. lesion closely matches a region of abrupt change in neuronal survival: photomicrographs of these two nearby regions were shown in panels $\mathrm{G}$ and $\mathrm{H}$ of Figure 6 . In the long-surviving ear, there is nearly complete neuronal degeneration, even in the apical half of the cochlea and despite the fact that there is significant survival of the IHC-supporting cells. Quantitative neuronal analyses were done for the peripheral axons only; however, qualitative analysis suggested that the survival of spiral ganglion cells was comparable (see images in Fig. 6).

To examine the relationship between supporting cell survival and neuronal survival more quantitative-

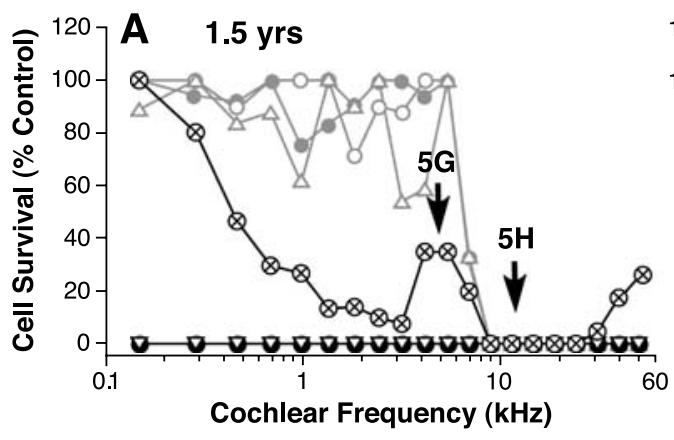

FIG. 7. Patterns of hair cell, supporting cell, and neuronal degeneration in two kanamycin-treated cat cochleas: one with a 1.5-year posttreatment survival (A) and one with a 5.5-year posttreatment survival (B). Symbol key in B applies also to A. Hair cell and supporting cell counts and neuronal survival estimates are

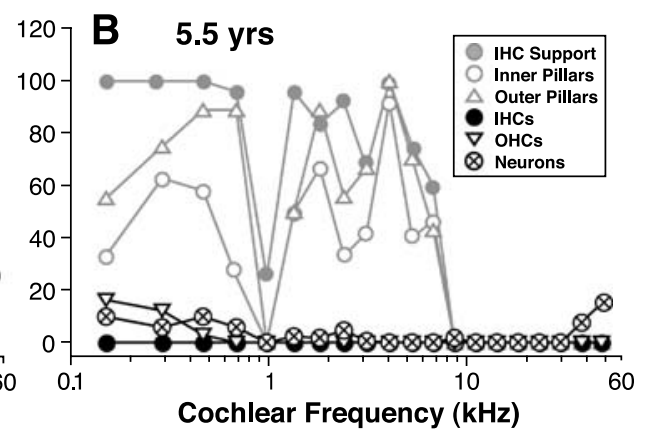

averaged over bins corresponding to $5 \%$ of cochlear length. Arrows in $\mathbf{A}$ labeled $5 \mathrm{G}$ and $5 \mathrm{H}$ refer to the micrographs taken from these two adjacent cochlear regions which appear in Figure 5. Cochlear length is converted to frequency according to a map derived by intracellular neuronal labeling (Liberman 1982a,b). 


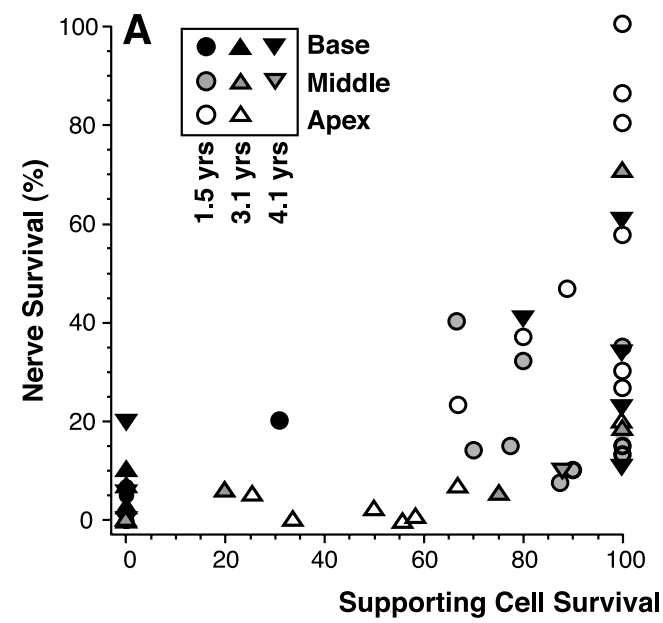

FIG. 8. Correlation between survival of supporting cells in the IHC area and the survival of auditory nerve peripheral axons, considering only regions from kanamycin-treated ears in which all IHCs are destroyed. Data are plotted separately for cochleas with posttreatment survival of 1.5 to 4.2 years (A) vs. 5.5 years (B). Data for each of the four survival times represent observations from two cochleas from one animal. Points in both panels are derived from data such as

ly, data from all aminoglycoside-treated ears in the present study are plotted in Figure 8. These scatterplots compare fractional neuronal survival to fractional IHC supporting-cell survival, considering only those cochlear regions in which all IHCs were missing. For all six cochleas from ears with survival times of $1.5,3.1$, or 4.1 years (Fig. $8 \mathrm{~A}$ ), there was enhanced neuronal survival in regions where IHC supporting cells remained. Although the correlation is not a tight one, significant neuronal survival was only seen when there was $>50 \%$ supporting cell survival, and when $<50 \%$ of supporting cells remained, the neuronal survival was never greater than $25 \%$. As for the carboplatin ears, the most robust neuronal survival was in the apex (unfilled symbols): at 1.5-year survival, some regions with IHC-supporting cells present showed no neuronal loss despite the total loss of IHCs. In contrast, at the 5.5-year survival (panel B), there was very little neuronal survival, despite the maintenance of a large number of supporting cells in the apical half of the cochlea. These data suggest that the presence of supporting cells in the absence of IHCs can delay, but possibly not prevent, the ultimate loss of afferent neurons.

\section{Auditory nerve section-primary neuronal degeneration and reinnervation}

Surgical section of the central axons of the auditory nerve leads to retrograde degeneration of type-I spiral ganglions and their central and peripheral axons (Spoendlin 1975). In the present study, the

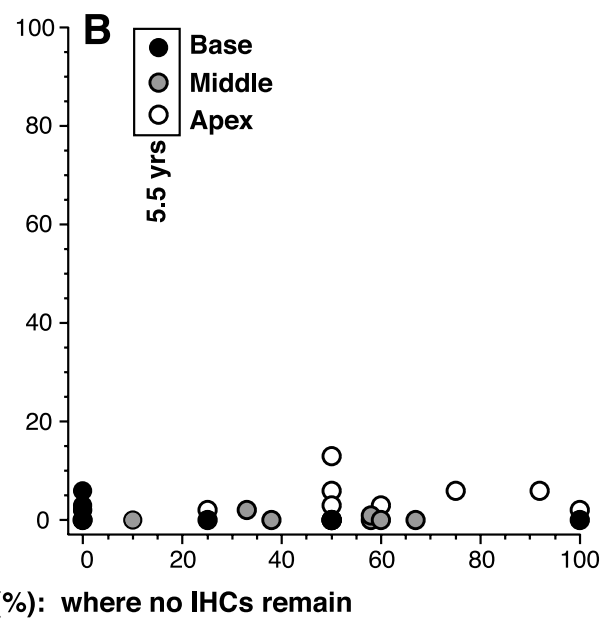

those shown in Figure 6, except that estimates of fractional survival for both cell types are averaged over bins corresponding to contiguous $10 \%$ segments of cochlear length. Data from basal, middle, and apical turns of the cochlea are plotted with different symbols (see key). Data from the basalmost $10 \%$ are excluded from both plots.

auditory nerve was cut unilaterally in nine cats with posttreatment survival times from 45 to 413 days. The degree and extent of neuronal degeneration was variable, as expected, given that the cuts were generally restricted to the caudal half of the nerve bundle, both to minimize the risk of interrupting the cochlear blood supply and to minimize involvement of vestibular nerves which exit the internal auditory meatus more rostrally. Loss of hair cells or supporting cells was only significant in one case, with a survival time of 69 days: it is likely that the cochlear blood supply was interrupted in this case.

The case with the longest survival time also showed the most complete deafferentation. It also showed the maximal involvement of the vestibular nerve; thus, it probably had the most complete initial cut. Photomicrographs in Figure 9 show massive loss of spiral ganglion cells as well as peripheral axons in the osseous spiral lamina (compare panels A and B). They also show that the overall architecture of the organ of Corti is normal, even 1.1 years after deafferentation. The cytocochleogram and neuronal counts for this case are shown in Figure 10: Apart from scattered IHC loss in the basal turn, all hair cells and supporting cells are present throughout the cochlea.

High magnification views of the IHC area reveal a pronounced difference in the pattern of innervation. In the control ear of this deafferented cat, a nest of unmyelinated type-I afferent terminals is visible at the base of the IHC (dotted circle in panel C), as well as a single terminal contacting the side of the IHC. In the deafferented ear, the IHC is flanked on both sides by 


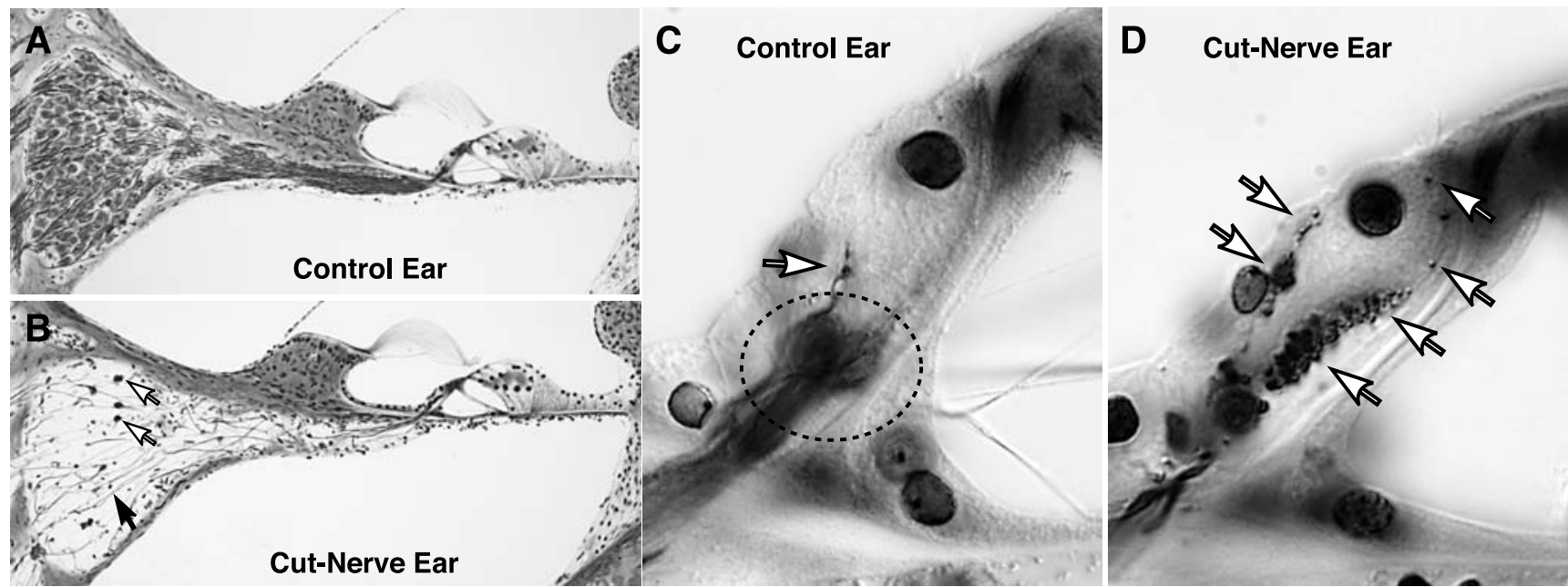

FIG. 9. Photomicrographs illustrating the cochlear duct (B) and IHC (D) area of a cat with 1.1-year survival from a surgical section of the auditory nerve. A, B Low-power micrographs of silver-stained sections through the cochlear duct: black arrow in $\mathbf{B}$ shows a fiber that traverses the spiral ganglion region without a cell body. White arrows point to two remaining spiral ganglion cells. C, D High- power views of the IHC area in the same sections shown in $\mathbf{A}$ and $\mathbf{B}$. Dotted circle in $\mathbf{C}$ indicates cluster of radial afferent terminals under the normal IHC; arrow in $\mathbf{C}$ points to one terminal contacting the side of the IHC. Arrows in D point to spiraling fibers which appear to be reinnervating the denervated IHC.

fibers seen in normal vestibular and facial nerves. Single-fiber recording from the auditory nerve trunk just before tissue harvesting revealed many fibers which generated action potentials in response to electric shocks delivered at the round window; however, none of these fibers had spontaneous activity or responses to sound.

\section{DISCUSSION}

Primary vs. secondary degeneration

Numerous studies, in both animal models and human temporal bones, have investigated the time course, pattern, and degree of cochlear neural degeneration in cases of hair cell loss due to acoustic injury (Bohne and Harding 2000; Liberman and Kiang 1978) or ototoxic drugs, notably aminoglycoside antibiotics (e.g., kanamycin, neomycin and amikacin; Koitchev et al. 1982; Leake and Hradek 1988; Lenoir et al. 1999; McFadden et al. 2004; Xu et al. 1993) or chemotherapeutic agents (such as carboplatin; Wang et al. 2003).

Loss of hair cells typically occurs before loss of neural elements. Hair cell loss after acoustic injury or massive doses of ototoxic drugs can be complete (at least within localized cochlear regions) within 1-3 days after the treatment, whereas the disappearance of peripheral axons requires a few weeks to reach comparable degrees of severity and the loss of spiral ganglion cells can take 1-2 months to appear (Leake and Hradek 1988; Liberman and Kiang 1978;
FIG. 10. Pattern of hair cell, supporting cell, and neuronal degeneration in one cat cochlea with a 1.1-year posttreatment survival from surgical section of the auditory nerve. All other conventions for data display are as described for Figure 6. 
McFadden et al. 2004). This progression of cell death has led to concept that hair cells are the primary targets of the insult and that the loss of neurons is secondary to loss of trophic support from the hair cells. On the other hand, there is evidence of early changes in neuronal terminals and cell bodies in the hours and days after these cochlear insults. Indeed, after carboplatin treatment, ultrastructural changes are seen in neural elements prior to those in hair cells (Wang et al. 2003). Similarly, in acoustic injury, dramatic swelling of afferent terminals can be seen under IHCs immediately after exposure, at a time when hair cell changes are hard to discern (Liberman and Mulroy 1982; Robertson 1983; Spoendlin 1971).

Until the precise mechanisms of action of these cochlear insults are better understood, there can be no definitive statement as to which effects are primary and which are secondary. Furthermore, there are likely signaling feedback loops among the cell types in the cell death cascade within this sensory epithelium such that the primary/secondary distinction may not be a particularly useful one.

Nevertheless, there is widespread agreement that, in cases of acoustic injury or ototoxic drugs, dramatic neuronal loss is typically seen in regions of dramatic IHC loss (Johnsson 1974b; Leake and Hradek 1988; Liberman and Kiang 1978; Zimmermann et al. 1995). Conversely, dramatic cell loss in the organ of Corti does not occur after near-total loss of cochlear neurons, even 1 year after nerve section (Fig. 10). Correspondingly, in human ears with Mohr-Tranebjaerg syndrome, there is significant neuronal loss without associated loss of cells within the organ of Corti (Merchant et al. 2001). Also, ouabain-induced degeneration of the spiral ganglion in the gerbil does not lead to hair cell loss or dysfunction (Schmiedt et al. 2002), although survival times beyond a few weeks have not been studied. These observations are consistent with the view that hair cells in the adult ear do not need an afferent innervation to survive, whereas cochlear afferent neurons require signaling interactions with cells in the sensory epithelium to remain viable.

The observation that IHC survival is most important to the survival of spiral ganglion cells is consistent with the fact that type-I neurons make synaptic contact with IHCs and comprise $95 \%$ of the cochlear nerve (Kiang et al. 1982b). However, if synaptic contact with IHCs were the only factor determining neuronal survivability, then fractional loss of IHCs should equal fractional loss of type-I peripheral axons, since these axons make synaptic contact with only a single IHC (Liberman 1980). The analysis in Figure 5 suggests that survival of a small percentage of IHCs can support the survival of a larger $(\sim 3 \times)$ percentage of neurons, even for years after cochlear insult, in regions where supporting cells survive. Several views of this relationship can be suggested: (1) that an IHC can release signals that affect the survival of nearby neurons which it does not contact; (2) that, after IHC loss, axons stripped of synaptic contact sprout to connect to surviving IHCs; or (3) that there are signaling interactions among IHCs and supporting cells such that surviving IHCs induce increased synthesis/release of trophic factors (e.g., neurotrophins) from surrounding supporting cells. It is not clear whether any, or all, of these occur; however, there are reports that peripheral axons resprout after hair cell loss from either acoustic overexposure (Strominger et al. 1995) or aminoglycoside-induced hair cell death (Backus et al. 1987; Tange and Huizing 1980).

\section{Role of supporting cells in neuronal survival: comparison to other studies}

An apparent correlation between supporting cell survival and neuronal survival has been noted in human temporal bone studies. For example, Johnsson (1974b) states that "it is not at all uncommon to find large portions of Corti's organ completely devoid of hair cells together with a seemingly intact nerve network in the osseous spiral lamina. Undoubtedly, the supporting elements effectively prevent or delay the nerve degeneration" (Johnsson 1974b, p. 299). Similar statements are made in other temporal bone studies (Johnsson et al. 1990; Suzuka and Schuknecht 1988) However, only one report (Suzuka and Schuknecht 1988) quantifies supporting cell survival along with hair cells and neurons. One case in that report is particularly relevant: an individual with 8-month survival from neomycin deafness shows loss of all IHCs and OHCs throughout the cochlea; inner pillar cells survived only in the apical half of the cochlea, and only in the apex was there significant neuronal survival.

Further evidence for a supporting cell role in neuronal survival is provided by animal work. Two studies on aminoglycoside-induced destruction of hair cells in cat report that long-term supporting cell survival and neuronal survival are greater in the apical than the basal half of the cochlea (Leake and Hradek 1988; Xu et al. 1993). The Leake study also notes a more localized correlation, i.e., patches of supporting cell loss associated with patches of neuronal loss, in regions completely devoid of IHCs. As in the present study, Leake and Hradek examined one set of cochleas at each of several survivals (i.e., 1, 3 , and 4 years). At 1 year, they report close to $100 \%$ neuronal survival in apical regions, if supporting cells remained after IHC loss; however, neuronal survival 
dropped to near zero by 3 years. In the present study, there was significant survival of apical neurons without IHCs until 4.1-year posttreatment, but not in the 5.5-year survival case. Thus, both studies agree that neuronal survival drops precipitously 3-5 years after IHC loss, even if supporting cells remain. Conversely, when supporting cells and IHCs degenerate early in the process, neuronal death proceeds quite rapidly. Salvi and coworkers, in a chinchilla model of gentamicin damage, found that spiral ganglion loss was essentially complete by $2-4$ months, in both apical and basal turns, when the supporting cells were also destroyed (McFadden et al. 2004).

The observation that supporting cell and neuronal survival tend to be greater in apical cochlear regions can be viewed in two ways: (1) that supporting cell survival directly enhances neuronal survival or (2) that survival of both cell types is enhanced due to some other apex-base gradient. The quantitative analysis in Figure 8A suggests that survival of supporting cells per se is important: although neuronal survival is generally better in the apex, basal, and middle regions, supporting cell survival also enhanced the survival of neurons. On the other hand, there is ample evidence for apex-to-base gradients for important signaling molecules (Judice et al. 2002). Furthermore, this report (Figs. 9 and 10) and previous reports (e.g., Spoendlin and Suter 1976) have noted a reinnervation of deafferented IHCs that is particularly robust in the cochlear apex. Such an apparent attraction of regenerating axons to the apical turn may be related to the increasing gradient of neurotrophin expression from base to apex (Sugawara et al., unpublished data).

\section{Signaling pathways involved in supporting-cell/} neuronal interactions

In the central nervous system, neurons are surrounded by glia, which provide trophic support necessary for neuronal survival under normal conditions and after injury (Cui et al. 2001; Reddy et al. 2003). In the organ of Corti, the unmyelinated terminals of cochlear afferents are ensheathed by supporting cells. These supporting cells, although derived from different embryonic cell types than glia, share expression patterns, e.g., GFAP (Rio et al. 2002), vimentin (Anniko et al. 1986), S100 (Pack and Slepecky 1995), and erbB receptors (Stankovic et al. 2004).

In other peripheral nerves, disruption of the neuregulin-erbB signaling pathway leads in nonmyelinating Schwann cells to neuronal death (Chen et al. 2003). Spiral ganglion cells express neuregulin (NRG; Morley 1998), and cochlear-supporting cells express erbB receptors, rendering them responsive to neuregulin (Stankovic et al. 2004). These supporting cells, along with IHCs, also express the neurotrophin NT-3, which can increase neuronal survival when delivered exogenously after hair cell loss (Miller et al. 1997). Expression of dominant-negative erbB receptors in supporting cells, which blocks neuregulinerbB signaling, leads to postnatal degeneration of cochlear neurons without concomitant IHC loss and to a reduction in cochlear NT-3 expression (Stankovic et al. 2004). Thus, it is reasonable to hypothesize (1) that both IHCs and their supporting cells release neurotrophins in response to neuregulin released by spiral ganglion cells, (2) that loss of IHCs leads to some neuronal loss due to decreased NT-3 levels, and (3) that supporting cell survival can at least delay further neuronal degeneration by virtue of the neuregulin-mediated neurotrophin release by the supporting cells themselves.

Regardless of the precise mechanisms, the present study provides empirical evidence that supporting cells contribute to the survival of sensory neurons after cochlear injury. After either carboplatin or aminoglycosides, hair cell loss occurs within weeks or months; however, neuronal loss progresses for years. At all survivals, IHC loss is a major determinant of neuronal loss as expected since $95 \%$ of cochlear afferents contact only IHCs and since IHCs express neurotrophins. Nevertheless, a small percentage of surviving IHCs "supports" a large degree of neuronal survival, even $>5$-year posttreatment. The aminoglycoside data suggests that supporting cells are key to this survival without IHCs. However, supporting cell survival may only delay, rather than prevent, neuronal degeneration, as neuronal loss was virtually complete, even with supporting cell survival, after 5.5 years.

\section{ACKNOWLEDGMENTS}

This research was supported by grants from the NIDCD: RO1 DC 04820 and P30 DC05209.

\section{REFERENCES}

Anniko M, Thornell LE, Gustavsson H, Virtanen I. Intermediate filaments in the newborn inner ear of the mouse. ORL J. OtoRhino-Laryngol. Relat. Spec. 48:98-106, 1986.

Backus RM, Degroot JC, Tange RA, Huizing EH. Pathological findings in the human auditory system following long-standing gentamicin ototoxicity. Arch. Oto. Rhino. Laryngol. 244:69-73, 1987.

Bohne BA, Harding GW. Degeneration in the cochlea after noise damage: primary vs. secondary events. Am. J. Otol. 21:505-509, 2000.

Brown MC, Berglund AM, Kiang NYS, Ryugo DK. Central trajecto- 
ries of type II spiral ganglion neurons. J. Comp. Neurol. 278:581-590, 1993.

Chen S, Rio C, Ji RR, Dikkes P, Coggeshall Re, Woolf CJ, Corfas G. Disruption of erbB receptor signaling in adult non-myelinating Schwann cells causes progressive sensory loss. Nat. Neurosci. 6:1186-1193, 2003.

Cui W, Allen ND, Skynner M, Gusterson B, Clark AJ. Inducible ablation of astrocytes shows that these cells are required for neuronal survival in the adult brain. Glia 34:272-282, 2001.

Eldredge DH, Miller JD, Bohne BA. A frequency-position map for the chinchilla cochlea. J. Acoust. Soc. Am. 69:1091-1095, 1981.

Fekete DM, Roulller EM, Liberman MC, Ryugo DK. The central projections of intracellularly labeled auditory nerve fibers in cats. J. Comp. Neurol. 229:432-450, 1982.

GaceK RR, Rasmussen GL. Fiber analysis of the statoacoustic nerve of guinea pig, cat, and monkey. Anat. Rec. 139:455-463, 1961.

Johnsson L-G. Sequence of degeneration of Corti's organ and its first-order neurons. Ann. Otol. Rhinol. Laryngol. 83:294, 1974.

Johnsson LG. Sequence of degeneration of Corti's organ and its first-order neurons. Ann. Otol. Rhinol. Laryngol. 83:294-303, 1974.

Johnsson LG, Felix H, Gleeson M, Pollak A. Observations on the pattern of sensorineural degeneration in the human cochlea. Acta Oto-Laryngol., Suppl. 470:88-95; discussion 95-86, 1990.

Judice TN, Nelson NC, Beisel CL, Delimont DC, Fritzsch B, Beisel KW. Cochlear whole mount in situ hybridization: identification of longitudinal and radial gradients. Brain Res. 9:65-76, 2002.

Kiang NY, Rho JM, Northrop CC, Liberman MC, Ryugo DK. Haircell innervation by spiral ganglion cells in adult cats. Science 217:175-177, 1982.

Kiang NYS, Rho JM, Northrop CC, Liberman MC, Ryugo DK. Haircell innervation by spiral ganglion cells in adult cats. Science 217:175-177, 1982.

Koitchev K, Guilhaume A, Cazals Y, Aran JM. Spiral ganglion changes after massive aminoglycoside treatment in the guinea pig. Counts and ultrastructure. Acta Oto-Laryngol. 94:431-438, 1982.

Leake PA, Hradek GT. Cochlear pathology of long term neomycin induced deafness in cats. Hear. Res. 33:11-33, 1988.

Lenoir M, Daudet N, Humbert G, Renard N, Gallego M, Pujol R, Eybalin M, VAGO P. Morphological and molecular changes in the inner hair cell region of the rat cochlea after amikacin treatment. J. Neurocytol. 28:925-937, 1999.

Liberman MC. Morphological differences among radial afferent fibers in the cat cochlea: an electron microscopic study of serial sections. Hear. Res. 3:45-63, 1980.

Liberman MC. The cochlear frequency map for the cat: labeling auditory-nerve fibers of known characteristic frequency. J. Acoust. Soc. Am. 72(5):1441-1449, 1982a.

Liberman MC. Single-neuron labeling in the cat auditory nerve. Science 216:1239-1241, 1982b.

Liberman MC, Kiang NYS. Acoustic trauma in cats, cochlear pathology and auditory-nerve activity. Acta Oto. Laryngol. 358:5-63, 1978.

Liberman MC, Mulroy MJ. Acute and chronic effects of acoustic trauma: cochlear pathology and auditory nerve pathophysiology. In: Hamernik RP, Henderson D, Salvi R (eds) New Perspectives on Noise-Induced Hearing Loss. Raven. New York, pp 105-136, 1982.

Liberman MC, Chesney CP, KuJawa SG. Effects of selective inner hair cell loss on DPOAE and CAP in carboplatin-treated chinchillas. Auditory Neurosci. 3:255-268, 1997.

McFadden SL, Ding D, Jiang H, Salvi RJ. Time course of efferent fiber and spiral ganglion cell degeneration following complete hair cell loss in the chinchilla. Brain Res. 997:40-51, 2004.

Merchant SN, McKenna MJ, Nadol Jr. JB, Kristiansen AG,
Tropitzsch A, Lindal S, Tranebjaeizrg L. Temporal bone histopathologic and genetic studies in Mohr-Tranebjaerg syndrome (DFN-1). Otol. Neurotol. 22:506-511, 2001.

Miller JM, Chi DH, O'Keeffe LJ, Kruszka P, Raphael Y, Altschuler RA. Neurotrophins can enhance spiral ganglion cell survival after inner hair cell loss. Int. J. Dev. Neurosci. 15:631-643, 1997.

MORLEY BJ. ARIA is heavily expressed in rat peripheral auditory and vestibular organs. Mol. Brain Res. 54:170-174, 1998.

PACK AK, SLEPECKY NB. Cytoskeletal and calcium-binding proteins in the mammalian organ of Corti: cell type-specific proteins displaying longitudinal and radial gradients. Hear. Res. 91:119-135, 1995.

Reddy LV, Koirala S, Sugiura Y, Herrera AA, Ko CP. Glial cells maintain synaptic structure and function and promote development of the neuromuscular junction in vivo. Neuron 40:563-580, 2003.

Rio C, Dikkes P, Liberman MC, Corfas G. Glial fibrillary acidic protein expression and promoter activity in the inner ear of developing and adult mice. J. Comp. Neurol. 442:156-162, 2002.

ROBERTSON D. Functional significance of dendritic swelling after loud sounds in the guinea pig cochlea. Hear. Res. 9:263-278, 1983.

Schmiedt RA, Okamura HO, Lang H, Schulte BA. Ouabain application to the round window of the gerbil cochlea: a model of auditory neuropathy and apoptosis. J. Assoc. Res. Otolaryngol. 3:223-233, 2002.

Schuknecht HF. Pathology of the Ear. Harvard University Press. Cambridge, 1974.

Spoendun H. Primary structural changes in the organ of Corti after acoustic overstimulation. Acta Oto. Laryngol. 71:166-176, 1971.

Spoendin H. Retrograde degeneration of the cochlear nerve. Acta Oto-Laryngol. 79:266-275, 1975.

SpoenduIN H. Factors inducing retrograde degeneration of the cochlear nerve. Ann. Otol. Rhinol. Laryngol. 93:76-82, 1984.

Spoendin H, Suter R. Regeneration in the VIII nerve. Acta Oto. Laryngol. 81:228-236, 1976.

Stankovic KM, Rio C, Xia A, Sugawara M, Adams JC, Liberman MC, CORFAS G. Survival of adult spiral ganglion neurons requires erbB receptor signaling in the inner ear. J. Neurosci. 24(40): 8651-8661, 2004

Starr A, Picton TW, Sininger Y, Hood LJ, Berlin CI. Auditory neuropathy. Brain 119(Pt 3):741-753, 1996.

Strominger RN, BoHne BA, Harding GW. Regenerated nerve fibers in the noise-damaged chinchilla cochlea are not efferent. Hear. Res. 92:52-62, 1995.

Suzuka Y, Schuknecht HF. Retrograde cochlear neuronal degeneration in human subjects. Acta Oto-Laryngol., Suppl. 450:1-20, 1988.

Takeno S, Harrison RV, Ibrahim D, Wake M, Mount RJ. Cochlear function after selective inner hair cell degeneration induced by carboplatin. Hear. Res. 75:93-102, 1994.

Tange RA, Huizing EH. Hearing loss and inner ear changes in a patient suffering from severe gentamicin ototoxicity. Arch. Oto. Rhino. Laryngol. 228:113-121, 1980.

WANG J, Ding D, SALVI RJ. Carboplatin-induced early cochlear lesion in chinchillas. Hear. Res. 181:65-72, 2003.

WARR WB. Olivocochlear and vestibular efferent neurons of the feline brain stem: their location, morphology and number determined by retrograde axonal transport and acetylcholinesterase histochemistry. J. Comp. Neurol. 161:159-182, 1975.

Xu SA, Shepherd RK, Chen Y, Clark GM. Profound hearing loss in the cat following the single co-administration of kanamycin and ethacrynic acid. Hear. Res. 70:205-215, 1993.

Zimmermann CE, Burgess BJ, Nadol JB Jr. Patterns of degeneration in the human cochlear nerve. Hear. Res. 90:192-201, 1995. 